\title{
Development of a Simulator for Tomographic Images Generated by Radiation Transmission
}

\author{
Gevaldo L. de Almeida, Maria Ines Silvani, Rosanne C. A. A. Furieri, \\ Instituto de Engenharia Nuclear-CNEN, C.P. 68550, Ilha do Fundão, 21945-970 Rio de Janeiro, RJ, Brasil \\ Marcelo J. Gonçalves, and Ricardo T. Lopes \\ Universidade Federal do Rio de Janeiro, COPPE, Centro de Tecnologia, Bloco 6, 21945-970, Rio de Janeiro, RJ, Brazil
}

Received on 9 September, 2003

\begin{abstract}
A computer program to simulate tomographic images generated by transmitted radiation was developed. The algorithm uses a deterministic approach to generate the projections, which supply an existing image reconstruction software. A dispersion associated with the counting statistics is also incorporated into the algorithm, in order to simulate the influence of the detector efficiency and counting time on the final image quality. The detector resolution is also included in the algorithm by assuming a gaussian shape for its line spread function LSF. The program deals with cylindrical objects containing any desired number of cylindrical rods inside and requires their positions, dimensions and attenuation properties as input data. Images of such objects, acquired with a thermal neutron tomograph equipped with a position sensitive detector, have been compared with those simulated by the developed program in order to evaluate its ability to reproduce those images.
\end{abstract}

\section{Introduction}

Computer Assisted Tomography - CAT is a growing branch of non-destructive assay applied in technology, engineering and medical examinations. A good review of the historical development of this methodology is presented by Brooks and Di Chiro [1] who also addressed a comprehensive discussion of the mathematical basis for the techniques used for image reconstruction, its constraints and limitations. The recent development and incorporation [2] of a Position Sensitive Detector - PSD to a thermal neutron tomographic system has stimulated several related works $[3,4,5,6]$ devoted to explore the advantages and potential capabilities of an image acquiring system of this kind, classified as a $2^{\text {nd }}$ generation tomograph, for it no longer requires a translation of the test objects as in the $1^{\text {st }}$ generation ones.

Disregarding their generation, one cannot forecast the quality of the final image unless the Modulation Transfer Function - MTF for the system is known. However, to obtain this function, it is necessary to carry out measurements using a special test object constituted by an opaque mask, containing alternating slits and spaces that increase in spatial frequency. Since these objects are very expensive, an alternative way is to perform a Fourier transform of the Line Spread Function, henceforth referred as LSF [7]. This function is difficult and somewhat cumbersome to be directly measured, but it can be approximated by a numerical differentiation of the Edge Response Function - ERF, which is easier to be obtained, for it deals with higher count rates due to the lower collimation constraints. $[7,8]$.

Anyhow, none of the outlined options would avoid a certain amount of experimental work to get the MTF. Even so, one could only estimate the expected degradation of the acquired image.

Therefore, a program to simulate a tomographic system, forecasting what image quality should be expected, has been developed for use as a guide to plan experiments. It furnishes projections of an user specified test object, which are then unfolded by a reconstruction program Recpar2000 [9]. This approach would cut down experimental efforts, reducing costs and eventual radiological burden associated with them.

\section{Tomographic principles}

Whenever an object intercepts a broad radiation beam, it produces a projection on a detecting system, which contains information about its structure. Rotating the object by a given angle, a new projection is obtained providing additional information about its components. The unfolding of several projections through a proper algorithm would furnish a 3D image of the object. In CAT systems, a 2Ddetector or a set of small single detectors distributed over a surface, collects data that can be processed and reconstructed by electronic means. In 2D systems, the object intercepts a slab-like narrow beam producing a line-shaped projection on a $1 \mathrm{D}$ detector. The unfolding of these projections would then furnish a cross-section image of the object at a perpendicular plane to its rotation axis. These projections could also be obtained by several single detectors distributed along a line, or alternatively a single detector together with a point source. In the last case however, besides the rotation, the object, or the source-detector set, should be 
translated to generate the aimed projections.

According to the CAT approach, an infinite number of projections would reproduce perfectly the attenuating structure of the object. In practice however, such a perfection would never be reached because the detector finite resolution spoils the image, making a line source to appear like a more or less blurred image, as dictated by the LSF - a curve reminding a Gaussian function, with its FWHM representing the detector resolution.

The non-zero detector resolution affects the quality of the image, degrading its contrast and resolution due to the overlapping of the LSF tails. In practice, these parameters are further worsened due to beam divergence and its nonmonochromatic character. Moreover, neutron scattering (or scattering via Compton effect when gamma or X-rays are used as incident radiation) take place contributing to an additional degradation of the final image.

\section{Algorithm}

The algorithm used by the simulator treated in this work, assumes an ideally parallel and monochromatic radiation beam, suffering no scattering as it interacts with the object and the detector.

The average count-rate per position-channel produced by an undisturbed beam is the result of an infinite number of infinitely narrow beams reaching the whole extension of each channel. Under such ideal conditions only those beams hitting directly each channel would contribute to the count rate. In practice however, even if a very thin slab beam is used to simulate a line source, the finite resolution of the detector would spoil that ideal conception. Since the LSF can be fairly represented by a Gaussian function, any beam hitting the detector would be seen by the detector as a Gaussian-shaped source, with its tail invading all the position spectrum, and thus, contributing to the count-rate at any channel. The degree of this contribution would depend on how far the Gaussian epicenter is from each particular channel and from their widths.

Taking the case of a position sensitive detector, having a total active length $\mathrm{L}$ coupled to a multi-channel analyzer and submerged into an homogeneous perfectly parallel radiation beam, then the average count-rate $\bar{T}_{j}$ produced at each channel $\mathrm{j}$ would be expressed by,

$$
\bar{T}_{j}=\frac{A_{0}}{L . \delta} \int_{0}^{L} \int_{x_{j}}^{x_{j}+\delta} e^{-\left[\frac{x-c}{w}\right]^{2}} d x d c
$$

with,

$A_{0}=$ constant expressing the beam intensity.

$\delta=$ channel width.

$w=$ width of the Gaussian expressing the LSF.

$C=$ epicenter of the above Gaussian.

$X_{j}=$ left side abcissa of the $\mathrm{j}^{\text {th }}$ channel.

$X=$ position along the position spectrum.

When a rotating object with its axis perpendicular to the detector intercepts a beam, its intensity is reduced by the inner attenuating structure of the object, generating a different projection for each angle that the object assumes. Applying a coordinate system to the region covering the cross-section of the object, one can define a function $\mu_{k}(x, y)$ expressing the attenuation coefficient as a function of the coordinates. Due to the rotation, this function will be different for each angle, since a new element, having a different attenuation coefficient from the previous one, will occupy a given point $(x, y)$. This angle-dependent character is expressed by the index $k$. The average count-rate $\bar{T}_{k j}$ produced at the $j^{t h}$ channel by the radiation beam disturbed by the object varies for each projection $k$, and is expressed as

$$
\bar{T}_{k j}=\frac{A_{0}}{L . \delta} \int_{0}^{L} \int_{x_{j}}^{x_{j}+\delta} e^{-\int_{y_{1}(x)}^{y_{2}(x)} \mu_{k}(x, y) d y} e^{-\left[\frac{x-c}{w}\right]^{2}} d x d c
$$

an expression which differs from (1) only due to the attenuation term containing the variables and parameters as follows, $\mu_{k}(x, y)=$ attenuation coefficient for the point $(\mathrm{x}, \mathrm{y})$ at the $k^{t h}$ projection. $\mathrm{y}_{1}(\mathrm{x}), \mathrm{y}_{2}(\mathrm{x})=$ lower and upper integration limits respectively as shown in Fig. 1

The count-rates produced by equations (1) and (2) depend, among other parameters, on the integration limit $\mathrm{L}$, for a Gaussian never vanishes but decreases asymptotically. Those count-rates are integrated over the acquisition time interval to yield the average counts per position channel stored in the multi-channel analyzer as ordinates of the position spectrum.

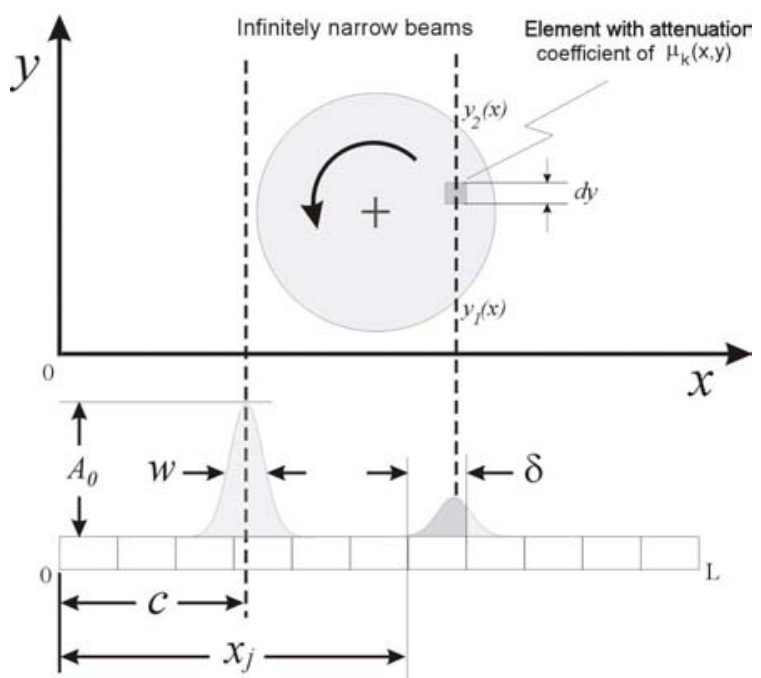

Figure 1. Diagram showing the relevant parameters and variables affecting the projection produced by a homogeneous parallel radiation beam.

The integration over time of those count-rates would result in a certain number of counts per channel, affected by a statistical fluctuation dictated by the Poisson distribution. This effect has been incorporated to the algorithm. 


\section{Effects of detector resolution and total counts on simulated images}

In order to evaluate the effects of detector resolution and total counts on the images reconstructed from projections produced by the simulator, a cylinder with sets of thin inserts was used.

The cylinder and the rods are made of hypothetical materials with attenuation coefficients of $0.1 \mathrm{~mm}^{-1}$ and 0.5 $\mathrm{mm}^{-1}$ respectively for the incoming radiation, disregarding its nature and energy. The purpose of this first test was to verify the soundness of the simulator. Within this frame, the nature and energy of the incident radiation do not play any role as long as they produce similar attenuation maps.

The responses of the simulator - for a channel width $\delta \mathrm{gf}$ $0.08 \mathrm{~mm}$ - to changes in the detector resolution and total counts are shown in Fig. 2. A degradation of the image resolution (merging dots) and contrast (changes of color) are observed as the detector resolution decreases as it should be theoretically expected. The decrease of contrast is caused by the overlapping of the LSF tails which grows with $w$.

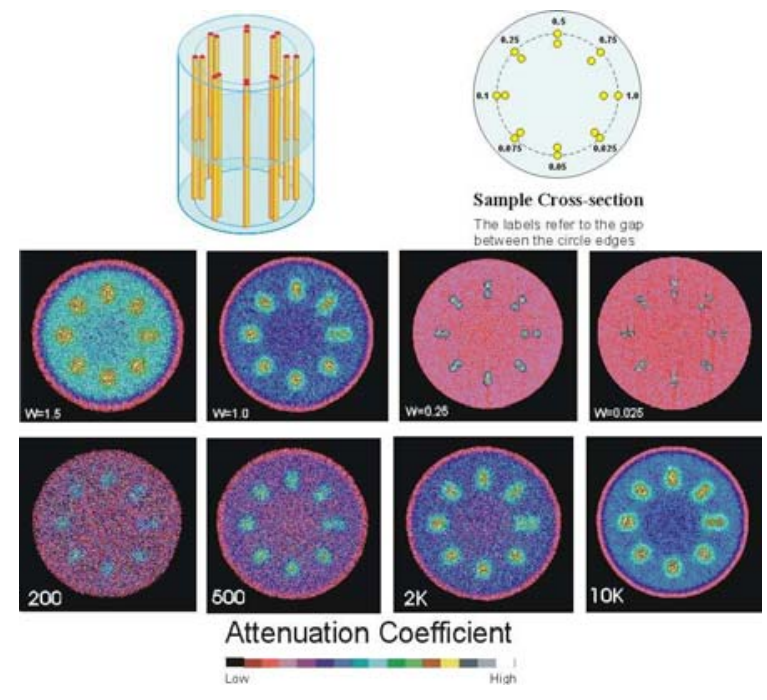

Figure 2. Response of the simulator to changes in the detector resolution $w$ (top) and total counts per channel (bottom).

Although not promptly recognizable - since the reconstruction software uses a color scale that differs from the natural and intuitive rainbow sequence - a careful observation shows that for $\mathrm{w}=1.5$ the rods and the cylinder exhibit the colors yellow and green respectively. For $w=0.25$ these colors are respectively gray and pink, which, according to the color scale are more distant from each other than the pair yellow-green.

When the total count increases, the image flickering diminishes and the merging of single features are better observed, but the image resolution remains the same, as in a real system.

\section{Materials and methods}

The tomographic system used to acquire the images and compare them with those simulated ones is schematized in Fig. 3. The Argonauta reactor at the Instituto de Engenharia Nuclear (IEN-CNEN-Brazil) has been utilized as the source of thermal neutrons, furnishing a flux of $4.5 \times 10^{5} \mathrm{ncm}^{-2} \mathrm{~s}^{-1}$ at the energy range $0.005-0.1 \mathrm{eV}$. A $40 \mathrm{~mm}$-deep honeycombtype collimator (hexagon side $=2 \mathrm{~mm}$ ) made of a $40 \mu$ thick gadolinium foil was used to improve the beam parallelism. The test object and the detector were placed at $18 \mathrm{~cm}$ and $26 \mathrm{~cm}$ respectively from the collimator front face. The PSD has an active length $\mathrm{L}$ of $80 \mathrm{~mm}$ and an active width of $5 \mathrm{~mm}$, as defined by its $2 \mathrm{~mm}$-thick aluminum window. The software Recpar 2000 uses the filtered back-projection approach to unfold the projections and reconstruct the images. Further details can be found elsewhere [3].

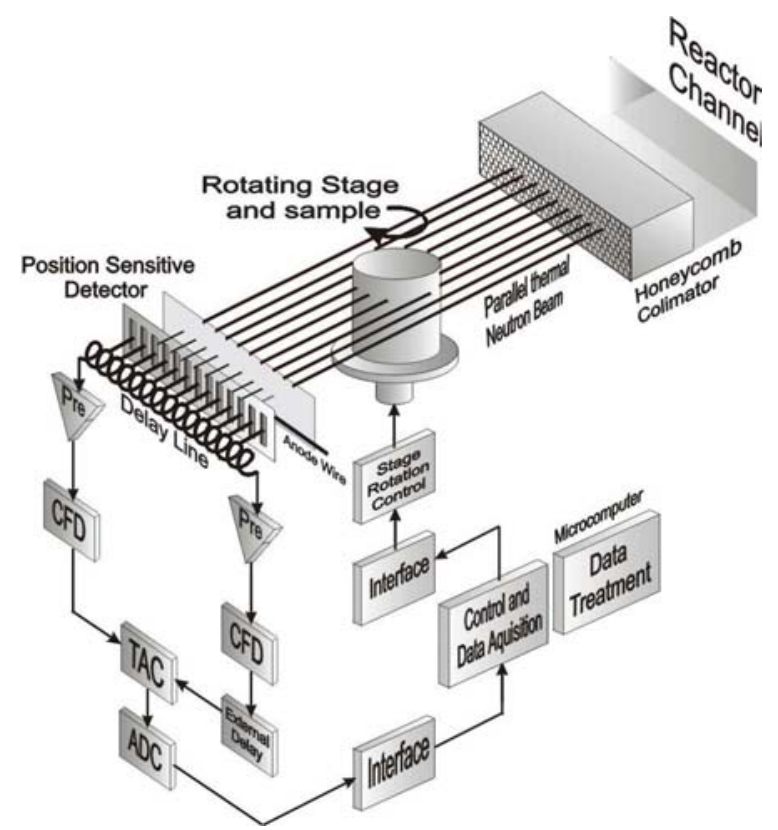

Figure 3. Scheme of the thermal neutron tomographic system used to acquire the images.

\section{Comparison with experimental re- sults}

While the simulator assumes a perfectly parallel neutron beam, suffering solely attenuation as it passes through the object, the real system deviates from these ideal condition due to more complex conditions and processes. Hence, the resolution of the simulator is dictated only by the resolution $w$ of its virtual detector, but in the real system, beam divergence and scattering processes contribute additionally to spoil the quality of the image. Therefore, it is not possible to infer the resolution of the real detector by comparing the simulated and real images. It is, however, feasible 
to estimate by visual inspection the resolution of the real system through that comparison, i.e. associating the $w$ of the simulator detector to the $w$ of the real system. After this approach, three different test objects have been used to compare the images generated by the simulator with those actually experimentally acquired.

Figure 4 focuses the capability of the real system to detect plastic materials within a teflon matrix and to differentiate them. Comparing the synthetic images with the real one, one can estimate the resolution of the system used to acquire it at about $1.3 \mathrm{~mm}$. As far as a visual inspection can infer, such a result is ratified by the similar contrasts achieved by the two kinds of images, both produced with about 4,000 counts per position channel. It can be furthermore recognized that the colors of the spots produced by the different materials are consistent with their attenuation coefficients and the color scale shown in Fig. 2.

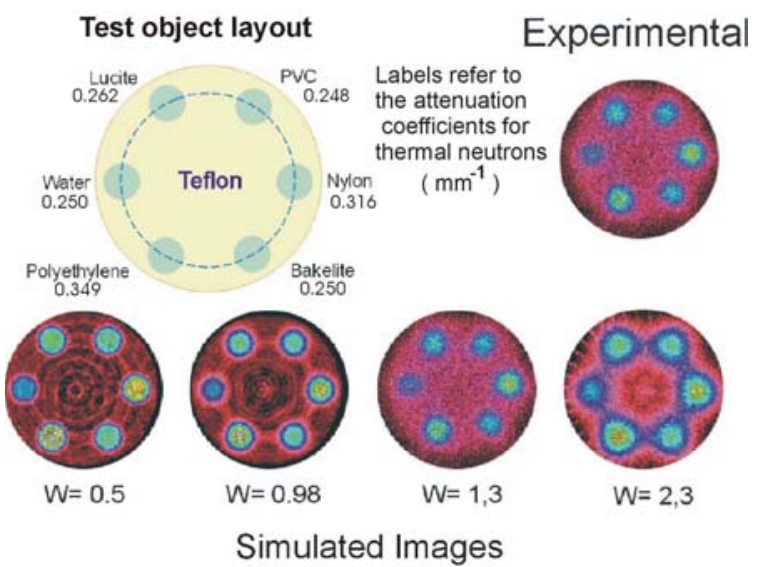

Figure 4. Simulated images under several detector resolutions $(w)$ compared with the experimental one. The test object is a $23 \mathrm{~mm}$ diameter teflon cylinder containing $4 \mathrm{~mm}$-diameter rods of different materials.

Images of the same teflon cylinder where the plastic rods have been replaced by metallic ones are shown in Fig. 5. One can conclude that the resolution of the system lies within the range of $1.3-1.7 \mathrm{~mm}$. It is worthwhile to point out that the two kinds of images (simulated and acquired) exhibit additional matching results in terms of contrast, as a visual inspection demonstrates. Indeed, in both of them aluminum is hardly detected, while lead is not detected at all, for its attenuation coefficient is very close to the teflon matrix, a behavior consistent with their attenuation coefficients for thermal neutrons.

The third test object used for comparison intended to assess the capability of the simulator to reproduce the performance of the real tomograph to detect and resolve small-size features.

A small-size feature requires a high attenuation coefficient for its detection, and to attain this condition, $1 \mathrm{~mm}$ thick cadmium wires have been introduced in orifices cast in a $23 \mathrm{~mm}$-diameter aluminum cylinder, after the scheme shown in Fig. 6, where the results are also presented.

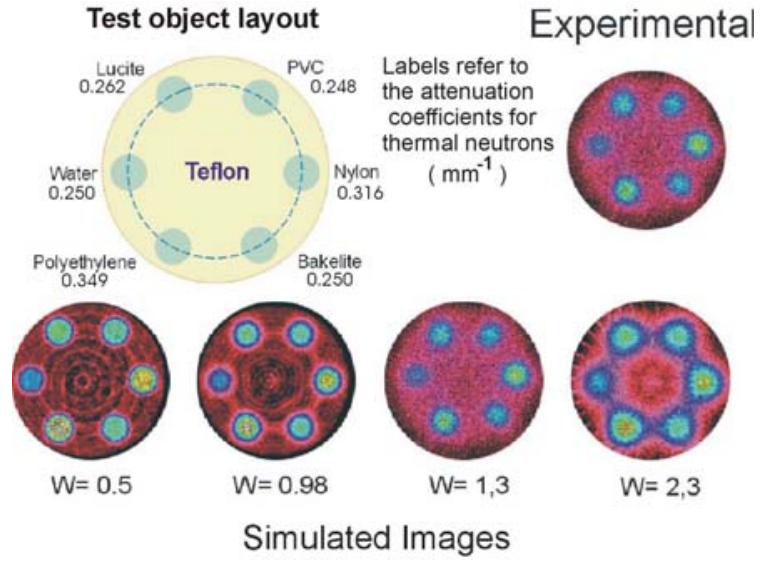

Figure 5. Same as Fig. 4, except for the rods materials.

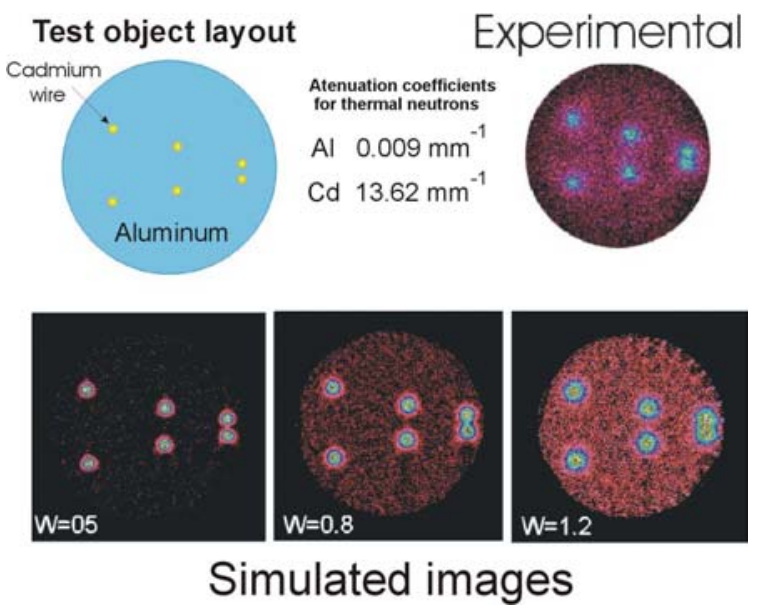

Figure 6. Simulated and experimental images of a $23 \mathrm{~mm}$-diameter $\mathrm{Al}$ cylinder containing $1 \mathrm{~mm}$-diameter $\mathrm{Cd}$ wires. The gap between the closest wires is $1 \mathrm{~mm}$.

Although the tomographic system could furnish a fair image of the test object, the simulator was unable to perform its task properly. The difficulty comes from the high incident-to-transmitted beam intensity ratio reaching $10^{17}$ for some projections generated by the simulator. The reconstruction software fails to handle such high numbers arising from the high attenuation coefficient of cadmium, for it was designed to deal with much lower values. Indeed, a tomographic image of acceptable quality is obtained when this ratio remains approximately within the range of $3-150$ [10], a feature which has been incorporated to the reconstruction software, making it incapable to process unexpected high ratios.

Therefore, to supply acceptable projections to the reconstruction software, the attenuation coefficient for the cadmium - given as input data to the simulator - had to be strongly reduced. Although such a reduction has obviously 
no physical meaning, it has been performed as a cross-check to assure that the difficulties really were caused by the high attenuation coefficient rather than by an eventual misconception in the architecture of the simulator. A reduction factor of about 20 was required to match the generated images with the real ones. Such a factor seems to be caused by beam divergence and neutron scattering. Indeed, both divergence and scattering would contribute to the average count-rate at a given channel, otherwise exposed only to those neutrons following a straight path intercepting the cadmium wire. The increased count-rate of the transmitted beam would then reduce the apparent attenuation coefficient for that particular path, reducing thus the incident-to-transmitted beam intensity ratio to a level acceptable by the reconstruction software.

Although the tomographic system could furnish a fair image of the test object, the simulator was unable to perform its task properly. The difficulty comes from the high incident-to-transmitted beam intensity ratio reaching $10^{17}$ for some projections generated by the simulator. The reconstruction software fails to handle such high numbers arising from the high attenuation coefficient of cadmium, for it was designed to deal with much lower values. Indeed, a tomographic image of acceptable quality is obtained when this ratio remains approximately within the range of $3-150$ [10], a feature which has been incorporated to the reconstruction software, making it incapable to process unexpected high ratios.

Therefore, to supply acceptable projections to the reconstruction software, the attenuation coefficient for the cadmium - given as input data to the simulator - had to be strongly reduced. Although such a reduction has obviously no physical meaning, it has been performed as a cross-check to assure that the difficulties really were caused by the high attenuation coefficient rather than by an eventual misconception in the architecture of the simulator. A reduction factor of about 20 was required to match the generated images with the real ones. Such a factor seems to be caused by beam divergence and neutron scattering. Indeed, both divergence and scattering would contribute to the average count-rate at a given channel, otherwise exposed only to those neutrons following a straight path intercepting the cadmium wire. The increased count-rate of the transmitted beam would then reduce the apparent attenuation coefficient for that particular path, reducing thus the incident-to-transmitted beam intensity ratio to a level acceptable by the reconstruction software.

\section{Conclusions}

The images reconstructed from simulator generated projections exhibit the expected features and behavior according to the changes in the detector resolution and counting statistics, demonstrating thus the soundness of the developed algorithm. For this simulator, the system resolution coincides with that of its detector, since this virtual device is the only component in the chain contributing to the degradation of the final image.

In a real tomograph however, the system resolution is poorer than its detector resolution due to beam divergence, alignment, scattering and other processes. Therefore, changing the resolution $w$ of the simulator until the synthetic image matches visually with that experimentally acquired would furnish an estimation of the tomograph resolution, namely $w$. Once this $w$ is known, the simulator could be used to plan experiments and to forecast the expected image quality prior to its acquisition, sparing thus experimental work and the associated radiological burden. The simulator didn't succeed in reproducing images of objects containing small-size features with extremely high attenuation properties, as the real tomograph did. This shortcoming has been imputed to its inability to take into account beam divergence and neutron scattering. The contribution of these processes to the reduction of the apparent attenuation coefficient of the test object is unknown. However, since the first one is simpler to be treated, and taking into account the fair results obtained, making the program capable of handling divergent beams is a further improvement and an worthwhile task.

In a real tomograph however, the system resolution is poorer than its detector resolution due to beam divergence, alignment, scattering and other processes. Therefore, changing the resolution $w$ of the simulator until the synthetic image matches visually with that experimentally acquired would furnish an estimation of the tomograph resolution, namely $w$. Once this $w$ is known, the simulator could be used to plan experiments and to forecast the expected image quality prior to its acquisition, sparing thus experimental work and the associated radiological burden. The simulator didn't succeed in reproducing images of objects containing small-size features with extremely high attenuation properties, as the real tomograph did. This shortcoming has been imputed to its inability to take into account beam divergence and neutron scattering. The contribution of these processes to the reduction of the apparent attenuation coefficient of the test object is unknown. However, since the first one is simpler to be treated, and taking into account the fair results obtained, making the program capable of handling divergent beams is a further improvement and an worthwhile task.

\section{References}

[1] R. A. Brooks and G. Di Chiro, Phys. Med. Biol. 21, 5689 (1976).

[2] M. I. Silvani, Computed Tomography with thermal neutrons and a Position Sensitive Detector, DSc. Thesis, COPPE/UFRJ-Brazil (2001). In Portuguese.

[3] M. I. Silvani, R. T. Lopes, E. F. Jesus, G. L. de Almeida, and A. F. Barbosa, Nucl. Instr. Meth. in Phys. Research A 505, 568 (2003).

[4] M. I. Silvani, R. T. Lopes, E. F. Jesus, G. L. de Almeida, A. F. Barbosa, and D. Braz, Conversion of a X-ray Position Sensitive Detector for use in Thermal Neutron Tomographic Systems, V International Topical Meeting on Industrial Radiation and Radioisotope Measurement Applications, Bologna, Italy (2002).

[5] M. I. Silvani, G. L. de Almeida, R. C. A. A. Furieri, R. T. Lopes, E. O. de Jesus, and A. F. Barbosa, Brazilian Journal of Physics, 33, 286 (2003). 
[6] M. J. Gonçalves, Optimization of a gaseous position sensitive detector for use in a thermal neutron tomographic system. M.Sc. Thesis, COPPE/UFRJ, Rio de Janeiro, RJ, Brazil (2003) In Portuguese.

[7] ASTM E 1441-95 and 1570-95a, Non-destructive Testing, Radiation Methods, computed Tomography, Guide for Imaging and Practice Examination, ISO/TC 135/SC5, N118 (1996).

[8] G. L. de Almeida, M. I. Silvani, R. T. Lopes, A Hybrid
Technique to Evaluate the Line Spread Function, VI ENAN, E10_142, Rio de Janeiro, Brazil (2002).

[9] Recpar2000, LIN-COPPE/UFRJ. Unpublished.

[10] R. Cesareo, A. Brunetti, R. T. Lopes, G. Galli, D. V. Rao, A. Castellano, G. E. Gigante, S. Mascarenhas, R. Robert, V. S. Filho, M. Gilardoni, H. P. da Silva, and P. Q. Colosso, $X$ and $\gamma$ ray tomography for non destructive material testing. SPIE Conference on developments in X-Ray Tomography II, Vol. 3772, Denver, Colorado, July (1999). 